\title{
Evaluation et amélioration de la qualité nutritionnelle des pâtes et des bouillies de maïs fermenté au Congo
}

\author{
Michel ELENGA, Joachim MASSAMBA, Simon C. KOBAWILA*, \\ Vheiye G. MAKOSSO et Thomas SILOU \\ Equipe Pluridisciplinaire de Recherche en Alimentation et Nutrition (Eprancongo) \\ B.P. 69, Faculté des Sciences, Université Marien Ngouabi, Brazzaville, Congo. \\ *Auteur correspondant, E-mail: kobawilasimon@yahoo.fr
}

\section{RESUME}

Le présent travail porte sur l'évaluation de la qualité nutritionnelle des pâtes et des bouillies traditionnelles dans les ateliers à Pointe-Noire, l'effet du sucre sur l'écoulement des bouillies et les effets des procédés modifiés sur la qualité nutritionnelle et l'écoulement des bouillies. L'évaluation de la qualité des bouillies traditionnelles dérivées des pâtes de maïs fermenté a montré que celles-ci présentent une faible densité énergétique de $52 \mathrm{kcal} / 100 \mathrm{~g}$ de MS et une médiocre teneur en protéines de 3,77 g/100g de MS et en lipides de 2,8 g/100g de MS. La consistance des bouillies reste indépendante du sucre incorporé. Afin d'améliorer les qualités nutritionnelles, des traitements technologiques basés sur l'ajout au procédé traditionnel de grains d'arachide et/ou de grains de maïs germé broyé ont été mis au point. L'analyse des pâtes et des bouillies issues des procédés modifiés montre une augmentation de la teneur en protéines à $12 \mathrm{~g} / 100 \mathrm{~g}$ de MS et de la teneur en lipides à $15 \mathrm{~g} / 100 \mathrm{~g}$ de MS pour la pâte et bouillie à base de mélange de maïs germé et de grains d'arachide décortiqués, la pâte et bouillie à base de mélange de maïs trempé et de grains d'arachide décortiqués, la pâte et bouillie à base de mélange de maïs trempé, de maïs germé et de grains d'arachide décortiqués. Les bouillies à base de maïs germé et de grains d'arachide décortiqués et à base de maïs trempé, de maïs germé et de grains d'arachide décortiqués se sont révélées nettement plus fluides que les trois autres qui sont apparues plus fluides que la bouillie obtenue à partir du procédé traditionnel. Le rôle des $\alpha$-amylases et des groupements polaires sur le comportement rhéologique des bouillies a été mis en évidence.

(C) 2009 International Formulae Group. All rights reserved.

Mots clés : Pâte fermentée, bouillie infantile, amylase, maïs germé.

\section{INTRODUCTION}

La malnutrition est la principale cause des 12 millions de décès d'enfants de moins de 5 ans (Caulfield et al., 2004). En République du Congo, près de 4470 enfants sont malnutris dont $26 \%$ souffrent de la malnutrition chronique, $6,6 \%$ sont émaciés et $14,5 \%$ ont une insuffisance pondérale (EDS
Congo, 2005). La forme de malnutrition protéino-énergétique la plus fréquente à Pointe-Noire où très peu d'études ont été faites sur l'alimentation infantile est le retard de croissance qui touche $13,7 \%$ des enfants de 0 à 71 mois, les prévalences de maigreur et d'insuffisance pondérale sont respectivement de $3,2 \%$ et $9,6 \%$ (Tchibindat, 1999). Cette 
malnutrition infantile apparaît vers l'âge de 3 mois, période qui correspond, dans la majorité des cas, à l'introduction de l'aliment de complément au lait maternel chez le nourrisson (Cornu et al., 1993). Pendant les six premiers mois de la vie, le système digestif du nourrisson se caractérise par une faible activité enzymatique, un faible volume de l'estomac de l'ordre de $30 \mathrm{~g}(\mathrm{ml}) / \mathrm{kg}$ de poids corporel (Sanchez-Grinan et al., 1992).

Les aliments de complément sont, dans la plupart des cas, préparés à partir des denrées locales, notamment des produits amylacés tels que le manioc et le maïs qui ne subissent aucun traitement enzymatique préalable et entrent seuls dans la confection des bouillies locales mis à part leur teneur élevée en sucre (Thomazic, 2003 ; NgomaMfoundou, 2001). Le maïs, sous forme fermentée, constitue la denrée la plus fréquemment utilisée dans la préparation des bouillies locales au Congo (Cornu et al., 1993 ).

La qualité nutritionnelle des pâtes et des bouillies de maïs fermenté à Pointe-Noire pourrait être identique à celle de l'ensemble $\mathrm{du}$ pays. L'évaluation de la qualité nutritionnelle des bouillies en rapport avec les procédés traditionnels peut être faite par le prélèvement des échantillons des grains de maïs, de pâte de maïs fermenté et de bouillie de maïs dans les ateliers de production.

Lorsque la mère prépare la bouillie pour son enfant, elle se trouve devant un dilemme: mettre moins de pâte ou de farine, ou, augmenter la quantité de matière sèche (Trèche, 2004). Ainsi, l'amidon des bouillies doit subir des traitements technologiques conduisant à augmenter leur fluidité.

Les modifications des procédés traditionnels pourraient être une solution onéreuse pour l'amélioration de la qualité nutritionnelle et/ou de la densité énergétique des bouillies. Des traitements technologiques susceptibles de modifier les propriétés de gonflement de l'amidon peuvent être utilisés pour améliorer la densité énergétique des bouillies, notamment les amylases industrielles (Cornu et al., 1995), les amylases de céréales germées (Traoré et al., 2007), la cuisson-extrusion (Mouquet et al., 2001, Mouquet et al., 2003). Le maïs germé à l'état humide pourrait être une solution adaptée à l'amélioration de la fluidité des bouillies. L'ajout aux céréales des légumineuses est souvent utilisé pour améliorer la qualité nutritionnelle des bouillies. Ainsi, les grains d'arachide conviennent comme l'aliment le plus adapté au contexte de Pointe-Noire. C'est dans ce cadre que s'inscrit la présente étude

Les traitements vont consister à enrichir en nutriments (lipides et protéines) les pâtes de maïs par l'ajout des grains d'arachide au cours de la fermentation, et, à appliquer au maïs fermenté l'hydrolyse enzymatique par le maïs germé humide, en vue d'adapter la consistance des bouillies à la physiologie digestive et aux besoins énergétiques du nourrisson.

Ainsi, le présent travail vise les objectifs suivants :

- évaluer dans le contexte de PointeNoire, la qualité nutritionnelle des pâtes et des bouillies de maïs fermenté en rapport avec les procédés traditionnels de leur production,

- évaluer l'influence de l'ajout de sucre sur l'écoulement des bouillies,

- déterminer l'effet de l'ajout des grains d'arachide sur la teneur en protéines, en lipides et en cendres des pâtes et bouillies de maïs fermenté,

- déterminer l'effet de l'ajout du maïs germé sur l'écoulement des bouillies.

\section{MATERIEL ET METHODES Matériel végétal}

Le matériel utilisé est constitué par les grains d'arachide et les grains de maïs de variété blanche utilisée par les productrices de la pâte de maïs fermenté (Thomazic, 2003). 
Méthodes

\section{Conditions de prélèvement des échantillons sur le terrain}

Des échantillons de grains de maïs, de pâte et de bouillies de maïs fermenté ont été prélevés au cours de deux enquêtes auxquelles à Pointe-Noire:

- La première est une enquête par questionnaires réalisée à domicile auprès d'un échantillon de femmes produisant habituellement de la pâte de maïs fermenté dans 50 ateliers représentatifs de la production et de la vente de ce produit.

- La deuxième enquête s'est déroulée auprès de 50 vendeuses des bouillies représentant la population générale des femmes réalisant la commercialisation de ce produit dans cette ville.

Méthodologie de prélèvement des échantillons dans les ateliers

Dans chaque atelier enquêté de production de la pâte de maïs fermenté, un échantillon de $500 \mathrm{~g}$ de grains de maïs est prélevé juste avant leur trempage en s'assurant que les grains prélevés sont représentatifs de l'ensemble du contenu du récipient en prenant les grains à la surface et au fond du récipient.

Après transformation des grains de mais en pâte de maïs fermenté, auprès de chaque productrice et dans chaque atelier enquêté, $500 \mathrm{~g}$ de pâte de maïs fermenté ont été prélevés et conditionnés dans les sachets étanches à l'eau et à l'air, en s'assurant que la pâte prélevée est représentative de l'ensemble de pâte produite en prenant la pâte à la surface et au fond du récipient. Les échantillons de grains de maïs et de pâte de maïs fermenté ont été prélevés et transportés dans une glacière jusqu'au laboratoire d'Epran en accueil au centre IRD de Pointe-Noire. Ils ont été conservés au congélateur pendant deux semaines avant les analyses.

Dans chaque lieu de commercialisation des bouillies, auprès de chaque vendeuse enquêtée, un échantillon de $200 \mathrm{~g}$ de bouillie a été prélevé. Les échantillons de bouillies prélevés ont été conditionnés dans les sachets étanches et transportés au laboratoire dans une glacière. Ces échantillons ont été conservés au congélateur pendant deux semaines avant les analyses.

Méthodologie de mise au point des procédés

La pâte de maïs fermenté a été reconstituée au laboratoire selon le schéma de la Figure 1 de façon à maîtriser toutes les étapes de la transformation. Cinq types de procédés ont été mis au point par modification du procédé traditionnel :

- la pâte à base de maïs germé (PG), le maïs germé (MG) a été produite selon le procédé décrit par Louembé et al. (2004);

- la pâte à base de mélange de maïs germé et de grains d'arachide préalablement séchés et décortiqués (MG/GA) aux proportions respectives de $4 \mathrm{~kg}$ et $1 \mathrm{~kg}$; la pâte à base de mélange de maïs germé et de maïs trempé (MG/MT) aux proportions respectives de $1 \mathrm{~kg}$ et $1 \mathrm{~kg}$;

- la pâte à base de mélange de maïs trempé et de grains d'arachide préalablement séchés et décortiqués (MT/GA) aux proportions respectives de $4 \mathrm{~kg}$ et $1 \mathrm{~kg}$;

- la pâte à base de mélange de maïs trempé, de maïs germé et de grains d'arachide préalablement séchés et décortiqués (MT/MG/GA) aux proportions respectives de $4 \mathrm{~kg}, 1 \mathrm{~kg}$ et $1 \mathrm{~kg}$.

Le mélange des ingrédients a été fait juste avant l'opération de broyage, pour chaque procédé modifié. Après cette étape, les différentes farines humides obtenues ont été traitées de la même manière que lors du schéma traditionnel de fabrication de la pâte de maïs fermenté.

\section{Méthodologie de préparation des bouillies au laboratoire}

Une quantité respective de 10, 20, 30, 40, 50, 60, 70, 80, 90, $100 \mathrm{~g}$ de chaque type de pâte produite au laboratoire a été diluée dans $180 \mathrm{ml}$ d'eau froide puis chauffée à l'aide d'une plaque électrique en position subterminale jusqu'au début de l'ébullition. 


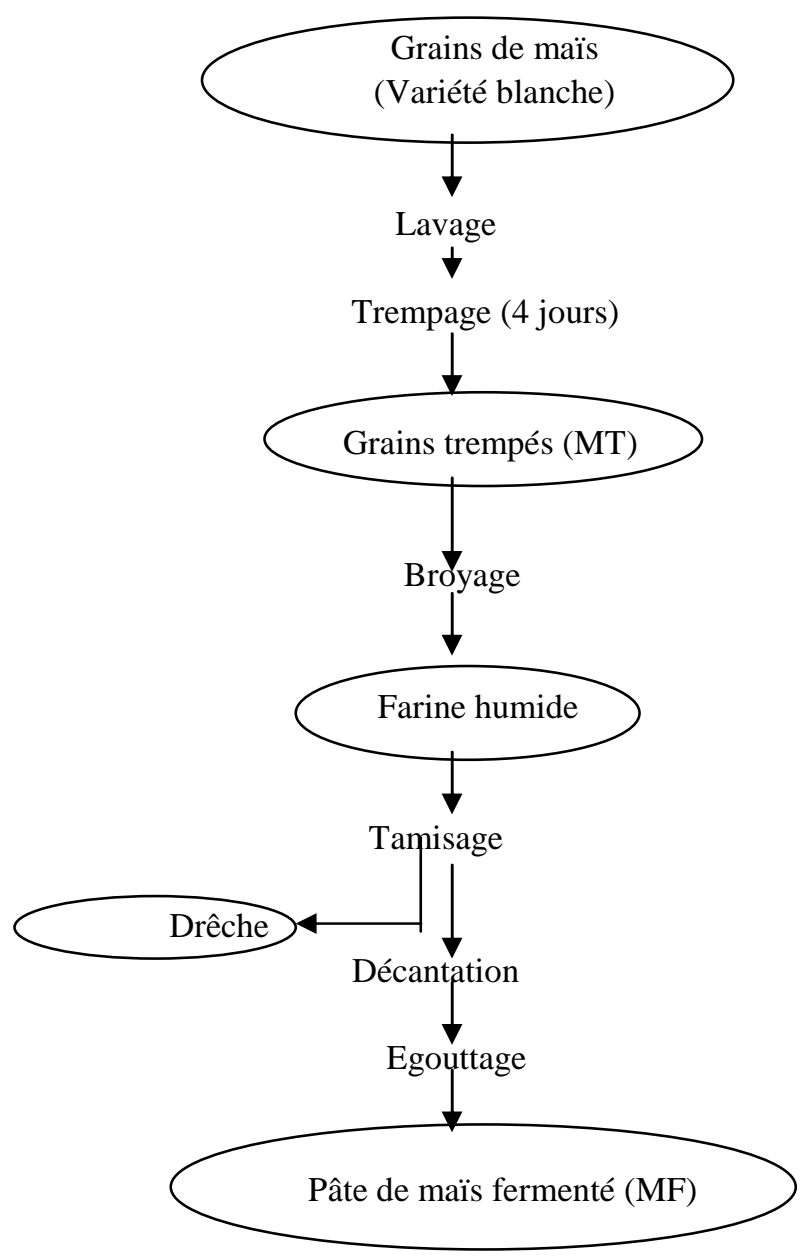

Figure 1 : Schéma de production des grains de maïs trempé (MT) et de la pâte traditionnelle de maïs fermenté (MF).

Pendant le lavage, les grains qui remontent à la surface de l'eau et ceux qui ont été attaqués par les insectes sont écartés. Pendant le de trempage, l'eau est renouvelée chaque jour en vue de se débarrasser des odeurs nauséabondes Pendant le broyage, les grains de maïs préalablement trempés et lavés, sont égouttés.

.Pendant le tamisage, la passoire retient les téguments qui sont élininés sous forme de drèche.

Pendant la décantation, la phase qui se dépose au fond du récipient est récupérée et le surnageant est éliminé. Pendant l'egouttage, l'eau est éliminée.

Le mélange a été retiré du feu et laissé au repos pendant $5 \mathrm{~min}$, pour permettre aux enzymes $\alpha$-amylases présentes d'agir ; puis, il a été remis au feu. La durée de cuisson a été fixée à $10 \mathrm{~min}$, après que le mélange ait atteint la température de $85^{\circ} \mathrm{C}$. La teneur en matière sèche des bouillies ainsi préparées a été déterminée. Ces valeurs ont permis de préparer des bouillies contenant 10,15,20, 25 $\mathrm{g}$ de matière sèche/100 $\mathrm{g}$ de bouillie et leurs vitesses d'écoulement ont été mesurées. Par ailleurs, afin de tester l'influence du sucre (saccharose) sur leur consistance, des bouillies ont été préparées au laboratoire, à quantité croissante de pâte de maïs fermenté traditionnel, en y ajoutant $16 \mathrm{~g}$ de sucre et leur vitesse d'écoulement a été comparée avec celle des bouillies sans sucre. 


\section{Méthodes d'analyses chimiques et physico- chimiques}

Le taux de matière sèche, les teneurs en protéines, en lipides, en cendres et en sels minéraux des échantillons ont été déterminés selon les méthodes de l'AOAC (1980) en triple essai.

Le taux de matière sèche a été déterminé par dessication à l'étuve à $105^{\circ} \mathrm{C}$ jusqu'à l'obtention d'un poids constant. La teneur en protéines a été déterminée par la méthode de Kjeldahl en utilisant un coefficient de conversion de l'azote en protéines de 6,25. La teneur en lipides a été déterminée par la méthode au soxhlet en utilisant l'hexane comme solvant d'extraction. Les cendres ont été déterminées par incinération au four à $530{ }^{\circ} \mathrm{C}$ et la teneur en calcium a été déterminée par spectrophotométrie d'absorption atomique.

La consistance des bouillies a été déterminée à l'aide d'un consistomètre de Bostwick qui permet de mesurer la vitesse d'écoulement des bouillies à $45{ }^{\circ} \mathrm{C}$ par la mesure de la distance parcourue en 30 secondes par le front de $100 \mathrm{ml}$ de bouillie. Le $\mathrm{pH}$ des échantillons des grains de maïs ou de la pâte de maïs en fermentation a été mesuré à l'aide d'un pH-mètre portable à sonde de type HI9214 Bioblock scientific. 100 $\mathrm{g}$ de farine issus des grains de maïs broyé ou $100 \mathrm{~g}$ de pâte de maïs en fermentation sont prélevés, homogénéisés dans $400 \mathrm{ml}$ d'eau distillée et filtrés à travers le papier filtre Whatman GF/A. Le filtrat obtenu a servi à la détermination du $\mathrm{pH}$.

\section{Analyse statistique des résultats}

Le calcul de la moyenne de chaque série de résultats ainsi que l'analyse statistique ont été réalisés à l'aide du logiciel Groph Pad InStat version 3.0. Le post test de Tukey était appliqué afin de déterminer si les différentes séries de valeurs étaient significativement différentes entre elles, au cas où le test ANOVA était significatif au moins au seuil $\mathrm{p}<0,05$.

\section{RESULTATS \\ Composition chimique des échantillons prélevés}

Le Tableau 1 présente l'évolution de la composition chimique ainsi que celle du $\mathrm{pH}$ au cours de la production des pâtes et des bouillies de maïs fermenté dans les ateliers.

Ce tableau montre une forte diminution du $\mathrm{pH}$, des teneurs en matière sèche et en cendres et, une faible diminution des teneurs en protéines et en lipides. La teneur en calcium connaît une légère augmentation.

\section{Composition chimique et caractéristiques physicochimiques des pâtes et des bouillies améliorées}

Le Tableau 2 présente la composition chimique ainsi que le pH des différentes pâtes issues des procédés modifiés.

La pâte traditionnelle de maïs fermenté prélevée dans les ateliers a une composition chimique et un $\mathrm{pH}$ quasiment identiques avec ceux de la pâte reproduite au laboratoire selon le procédé traditionnel.

En effet, ces deux produits ne présentent qu'une légère différence de teneur en matière sèche $(51 \%$ pour le produit original des ateliers contre $50,05 \%$ pour la pâte reconstituée au laboratoire). Ce résultat a légitimé l'utilisation de cette pâte préparée au laboratoire, comme témoin en référence à l'étude des pâtes modifiées préparées dans les mêmes conditions.

Trois des cinq pâtes issues des procédés modifiés, contenant de l'arachide (MT/GA, MT/MG/GA et MG/GA) présentent respectivement des teneurs en matière sèche supérieures à celles du témoin. Par contre, les teneurs en matière sèche des pâtes ne contenant pas les grains d'arachide (MG/MT et $\mathrm{PG}$ ) sont respectivement plus faibles que celle du témoin.

En ce qui concerne les protéines, leur teneur s'est améliorée respectivement pour les pâtes issues des procédés modifiés contenant de l'arachide (MT/GA, MG/GA, $\mathrm{MT} / \mathrm{MG} / \mathrm{GA})$. Elle va des valeurs respectives 
de $9,85 \mathrm{~g} / 100 \mathrm{~g}$ de MS, $11,88 \mathrm{~g} / 100 \mathrm{~g}$ de MS et $12,31 \mathrm{~g} / 100 \mathrm{~g}$ de MS contre 7,61 g/100g de MS pour le témoin. Par contre, les pâtes ne contenant pas de l'arachide (MG/MT, MT) ont présenté une légère diminution de leur teneur en protéines par rapport au témoin.

S'agissant de la teneur en lipides des trois pâtes renfermant de l'arachide (MT/GA, MG/GA et MT/MG/GA), elle a subi une augmentation très importante allant dans le sens de plus du quadruple par rapport au témoin.

Les deux pâtes issues des procédés modifiés sans apport d'arachide (MG/MT et PG) ont connu une baisse de teneur en lipides qui est passée respectivement de $2,8 \mathrm{~g} / 100 \mathrm{~g}$ de MS et 2,4 $\mathrm{g} / 100 \mathrm{~g}$ de MS contre 3,6 g/100g de MS pour le témoin.

Le Tableau 3 présente la composition chimique et le $\mathrm{pH}$ des bouillies à base de pâtes issues des procédés modifiés. Il montre que l'évolution de la composition chimique et celle des caractéristiques physico-chimiques des bouillies vont dans le même sens que celles des pâtes issues des procédés modifiés dont elles dérivent.

Le $\mathrm{pH}$ connaît une légère augmentation lors du passage des pâtes aux bouillies pour les mêmes raisons évoquées lors de l'étude des bouillies issues de la pâte fermentée traditionnelle.

Evolution de la consistance des bouillies avec ou sans sucre en fonction de la teneur en matière sèche

L'évolution de la consistance des bouillies de maïs fermenté avec ou sans sucre est donnée par la Figure 2. Cette figure montre que l'ajout de sucre lors de la cuisson de la bouillie de maïs fermenté comme cela se fait traditionnellement par les mamans, ne modifie quasiment pas l'allure de la courbe de l'évolution de la vitesse d'écoulement en fonction de la teneur en matière sèche.

L'incorporation du sucre aux bouillies, bien qu'elle en augmente l'appétence (goût), n'a aucun impact sur la densité énergétique des bouillies. Ce résultat pourrait par conséquent expliquer en partie la prévalence de la malnutrition observée dans le pays en général et à Pointe-Noire en particulier, ville dans laquelle notre étude est conduite.

\section{Consistance des bouillies dérivées de la pâte traditionnelle de maïs fermenté et celle des bouillies issues des pâtes des procédés modifiés}

Les vitesses d'écoulement des bouillies issues des procédés modifiés et celles des bouillies dérivées de la pâte traditionnelle de maïs fermenté sont présentées par la Figure 3. Ce graphique montre que les bouillies à base de MG/GA et à base de MT/MG/GA sont plus fluides que les trois autres (PG, MG/MT, MT/GA), lesquelles, à leur tour, sont apparues plus fluides que la bouillie obtenue à partir du procédé traditionnel.

\section{DISCUSSION}

La fermentation est une des vieilles et économiques méthodes de transformation des produits alimentaires, très utilisées dans les ménages dans les pays en voie de développement; Il s'agit des fermentations de types naturelles. Les résultats obtenus montrent une baisse du $\mathrm{pH}$ de 6,31 à 3,51 due à la formation d'acides organiques produits par la microflore lactique au cours des étapes fermentaires (trempage, tamisage, égouttage). Les travaux de Tou et al. (2006) montrent une diminution du $\mathrm{pH}$ lors de la fabrication du ben-saalga. Mensah (1997) rapporte que la fermentation de la pâte de maïs fermenté peut occasionner l'inhibition de certains microorganismes notamment les bactéries gram négative. Plusieurs auteurs (Muyanja et al., 2002 ; Oyewole, 1997 ; Nout et al., 1996, Louembé et al., 1996) pensent que la fermentation en abaissant le $\mathrm{pH}$ des produits à des valeurs inférieures à 4,0 limite le développement des entérobactéries et autres bactéries gram négative. 
Tableau 1: Composition chimique et caractéristiques physicochimiques des échantillons des produits dérivés des grains de maïs prélevés dans les ateliers urbains.

\begin{tabular}{lcccccc}
\hline $\begin{array}{l}\text { Produits } \\
\text { étudiés }\end{array}$ & $\begin{array}{c}\text { Matière } \\
\text { sèche } \\
(\mathbf{g} / \mathbf{1 0 0 g} \mathbf{~ d e} \\
\mathbf{M B})\end{array}$ & $\begin{array}{c}\text { Protéines } \\
\text { brutes } \\
(\mathbf{g} / \mathbf{1 0 0 g} \mathbf{~ d e} \\
\mathbf{M S})\end{array}$ & $\begin{array}{c}\text { Lipides } \\
\text { brutes } \\
\mathbf{( g / 1 0 0 g} \\
\mathbf{d e ~} \mathbf{M S})\end{array}$ & $\begin{array}{c}\text { Cendres } \\
(\mathbf{g} / \mathbf{1 0 0 g} \\
\mathbf{d e ~} \mathbf{M S})\end{array}$ & $\begin{array}{c}\text { Calcium } \\
(\mathbf{m g} / \mathbf{1 0 0 g} \\
\mathbf{d e ~} \mathbf{M S})\end{array}$ & pH \\
\hline Grains de maïs & $84,84 \pm 2,49$ & $9,66 \pm 1,18$ & $4 \pm 0,60$ & $1,47 \pm 0,11$ & $20 \pm 0,57$ & $6,31 \pm 0,32$ \\
$\begin{array}{l}\text { Pâte de maïs } \\
\text { fermenté }\end{array}$ & $-51,35 \pm 0,05^{* * *}$ & $-7,61 \pm 1,09^{* * *}$ & $-3,6 \pm 0,56^{* * *}$ & $-0,43 \pm 0,10^{* * *}$ & $23 \pm 1,00 \mathrm{~ns}$ & $-3,51 \pm 0,17^{* * *}$ \\
$\begin{array}{l}\text { Bouillie de } \\
\text { maïs fermenté }\end{array}$ & $-13,61 \pm 2,20^{* * *}$ & $-3,77 \pm 1,30^{* * *}$ & $-2,8 \pm 0,45^{* * *}$ & $-0,29 \pm 0,09^{* * *}$ & $+28 \pm 1,73^{* *}$ & $-3,84 \pm 0,13^{* * *}$ \\
\hline $\begin{array}{l}\text { Les valeurs sont les moyennes } \pm \text { Ecart type pour } \mathrm{n}=50 ; * * * \mathrm{p}<0,001 ; * * \mathrm{p}<0,01 ; \mathrm{ns} \mathrm{p}>0,05 \mathrm{p}=\text { probabilité } ; \mathrm{ns}=\text { non } \\
\text { significatif. }\end{array}$
\end{tabular}

Tableau 2: Composition chimique globale des pâtes issues des procédés modifiés.

\begin{tabular}{|c|c|c|c|c|c|}
\hline $\begin{array}{l}\text { Type de pâte } \\
\text { améliorée de } \\
\text { maïs fermenté }\end{array}$ & $\begin{array}{l}\text { Matière sèche } \\
\text { (g/100g de MB) }\end{array}$ & $\begin{array}{l}\text { Protéines brute } \\
\text { (g/100g de MS) }\end{array}$ & $\begin{array}{l}\text { Lipides } \\
\text { g/100g de } \\
\text { MS) }\end{array}$ & $\begin{array}{c}\text { Cendres } \\
\text { (g/100g de } \\
\text { MS) }\end{array}$ & pH \\
\hline $\begin{array}{l}\text { Maïs trempé } \\
\text { (Témoin) }\end{array}$ & $50.05 \pm 0,11$ & $7,61 \pm 0,36$ & $3,6 \pm 0,00$ & $0,43 \pm 0,01$ & $3,61 \pm 0,005$ \\
\hline PG & $-48,30 \pm 0,19 \mathrm{~ns}$ & $-6,94 \pm 0,01 \mathrm{~ns}$ & $-2,4 \pm 0,11 \mathrm{~ns}$ & $-0,20 \pm 0,01 \mathrm{~ns}$ & $+3,72 \pm 0,003^{* * *}$ \\
\hline MG/GA & $+51,21 \pm 0,49 \mathrm{~ns}$ & $+11,88 \pm 0,005^{*}$ & $+15 \pm 0,07^{* * * *}$ & $-0,35 \pm 0,005 \mathrm{~ns}$ & $+3,86 \pm 0,003^{* * *}$ \\
\hline MG/MT & $-49,03 \pm 0,19 \mathrm{~ns}$ & $-6,94 \pm 0,00 \mathrm{~ns}$ & $-2,8 \pm 0,05^{* * * *}$ & $-0,20 \pm 0,006 \mathrm{~ns}$ & $+3,72 \pm 0,003^{* * *}$ \\
\hline MT/GA & $+52,58 \pm 0,07^{\text {** }}$ & $+9,85 \pm 2,38^{*}$ & $+15,13 \pm 0,01^{* * *}$ & $+0,52 \pm 0,01 \mathrm{~ns}$ & $-3,60 \pm 0,011 \mathrm{~ns}$ \\
\hline MT/MG/GA & $+51,64 \pm 0,04 \mathrm{~ns}$ & $+12,31 \pm 0,003$ * & $+14,6 \pm 0,11^{* * * *}$ & $-0,35 \pm 0,005 \mathrm{~ns}$ & $+3,86 \pm 0,01^{* * *}$ \\
\hline $\begin{array}{l}\text { PG : pâte à base } \\
\text { MG/GA : la pâte } \\
\text { MT/GA : la pâte } \\
\text { MT/MG/GA : la } \\
\text { décortiqués } \\
\text { MT/MT : la pâte } \\
\text { MF : bouillie à b } \\
\text { Les valeurs sont }\end{array}$ & $\begin{array}{l}\text { grains de maïs germé } \\
\text { base de mélange de ma } \\
\text { base de mélange de ma } \\
\text { pâte à base de mélange } \\
\text { base de mélange de ma } \\
\text { se de pâte de maïs ferm }\end{array}$ & $\begin{array}{l}\text { aïs germé et de grains } \mathrm{d} \\
\text { iis trempé et de grains d } \\
\text { de maïs trempé, de } \mathrm{m} \\
\text { aïs germé et de maïs tre } \\
\text { entée produite au labora }\end{array}$ & $\begin{array}{l}\text { 'arachide préalablem } \\
\text { l'arachide préalablen } \\
\text { aaìs germé et de gre } \\
\text { mpé } \\
\text { toire }\end{array}$ & $\begin{array}{l}\text { ent séchés et décortiq } \\
\text { int séchés et décortic } \\
\text { ins d'arachide préala }\end{array}$ & $\begin{array}{l}\text { lés } \\
\text { ués } \\
\text { lement séchés et }\end{array}$ \\
\hline
\end{tabular}

Les résultats obtenus montrent que les procédés technologiques utilisés ont une incidence négative sur la valeur nutritionnelle et la densité énergétique de ces bouillies. Ces résultats confirment ceux obtenus par plusieurs études (Bouvier, 1992 ; Louembé et al., 1996 ; Louembé et al., 2003).
Lors de la transformation des grains de maïs fermenté en pâte de maïs fermenté, les substances en solution dans l'eau et les protéines seraient éliminées avec l'eau de trempage, de décantation et d'égouttage: ce qui entraînerait la diminution observée de la teneur en matière sèche, en cendres et en protéines. 
Tableau 3 : Composition globale et $\mathrm{pH}$ des bouillies dérivées des pâtes de maïs fermenté issues des procédés modifiés.

\begin{tabular}{llllll}
\hline $\begin{array}{c}\text { Type de bouillies } \\
\text { dérivées des pâtes } \\
\text { améliorées de maïs } \\
\text { fermenté }\end{array}$ & $\begin{array}{c}\text { Matière sèche } \\
(\mathbf{g} / \mathbf{1 0 0 g} \text { de } \mathbf{M B})\end{array}$ & $\begin{array}{c}\text { Protéines } \\
\text { brutes } \\
(\mathbf{g} / \mathbf{1 0 0 g} \text { de }\end{array}$ & $\begin{array}{c}\text { Lipides } \\
(\mathbf{g} / \mathbf{1 0 0 g} \mathbf{~ d e} \\
\mathbf{M S})\end{array}$ & $\begin{array}{c}\text { Cendres } \\
(\mathbf{g} / \mathbf{1 0 0 g} \mathbf{~ d e} \\
\mathbf{M S})\end{array}$ & $\mathbf{p H}$ \\
\hline PG & $10,35 \pm 0,01$ & $6,31 \pm 0,005$ & $2,8 \pm 0,05$ & $0,35 \pm 0,005$ & $4 \pm 0,00$ \\
MG/MT & $10,03 \pm 0,005^{* * *}$ & $5,69 \pm 0,005^{* * * *}$ & $2,4 \pm 0,15 \mathrm{~ns}$ & $0,35 \pm 0,011^{* *}$ & $4 \pm 0,00$ \\
MG/GA & $11,07 \pm 0,01^{* * *}$ & $8 \pm 0,01^{* * *}$ & $12,8 \pm 0,19^{* * * *}$ & $0,50 \pm 0,011^{* * *}$ & $4 \pm 0,00$ \\
MT/GA & $11,80 \pm 0,01^{* * *}$ & $11 \pm 0,02^{* * *}$ & $13,50 \pm 0,01^{* * *}$ & $0,55 \pm 0,01^{* * *}$ & $4 \pm 0,00$ \\
MT/MG/GA & $10,51 \pm 0,005^{* * *}$ & $12.03 \pm 0,01^{* * *}$ & $13,65 \pm 0,005^{* * *}$ & $0,55 \pm 0,006^{* * *}$ & $4 \pm 0,00$ \\
\hline
\end{tabular}

PG : bouillie à base de pâte de maïs germé

MG/GA : bouillie à base de pâte de maïs germé - grains d'arachide

MT/GA : bouillie à base de pâte de maïs trempé - grains d'arachide

MT/MG/GA : bouillie à base de pâte de maïs trempé - maïs germé - grains d'arachide

MT/MT : bouillie à base de pâte de maïs germé - maïs trempé

Les valeurs sont les moyennes \pm Ecart type pour $\mathrm{n}=3$; *** $\mathrm{p}<0,001 ; \mathrm{ns} \mathrm{p}>0,05 ; \mathrm{p}=$ probabilité.

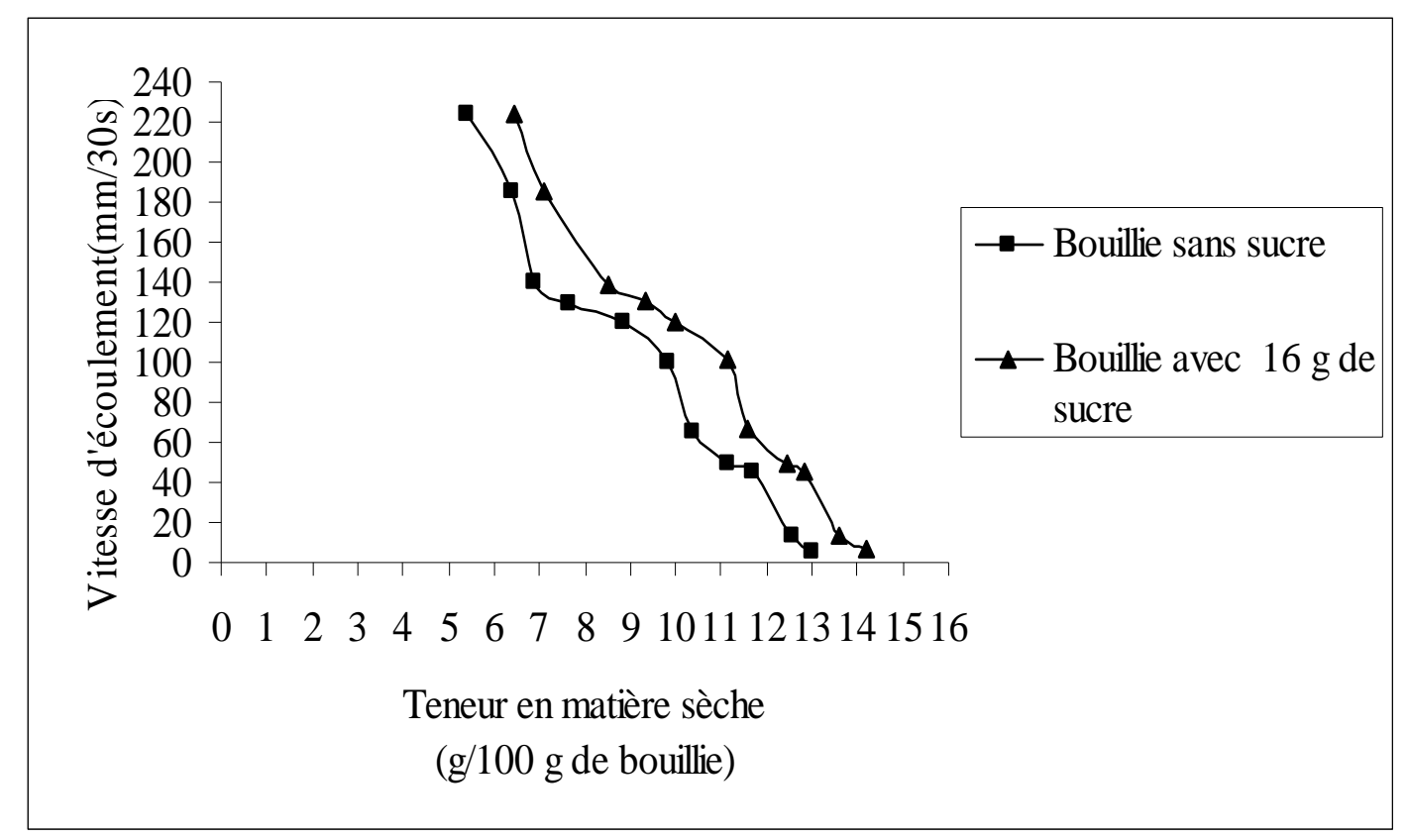

Figure 2: Evolution de la vitesse d'écoulement en fonction de la matière sèche des bouillies préparées avec ou sans sucre.

La teneur en protéines diminue de $21 \%$ au cours du procédé de transformation des grains de maïs en pâte de maïs fermenté comme l'ont souligné les travaux de Bouvier
(1992). La flore lactique en développement au cours de la fermentation utilise les protéines pour son métabolisme, ce qui contribue à la diminution de la teneur de ces protéines. 


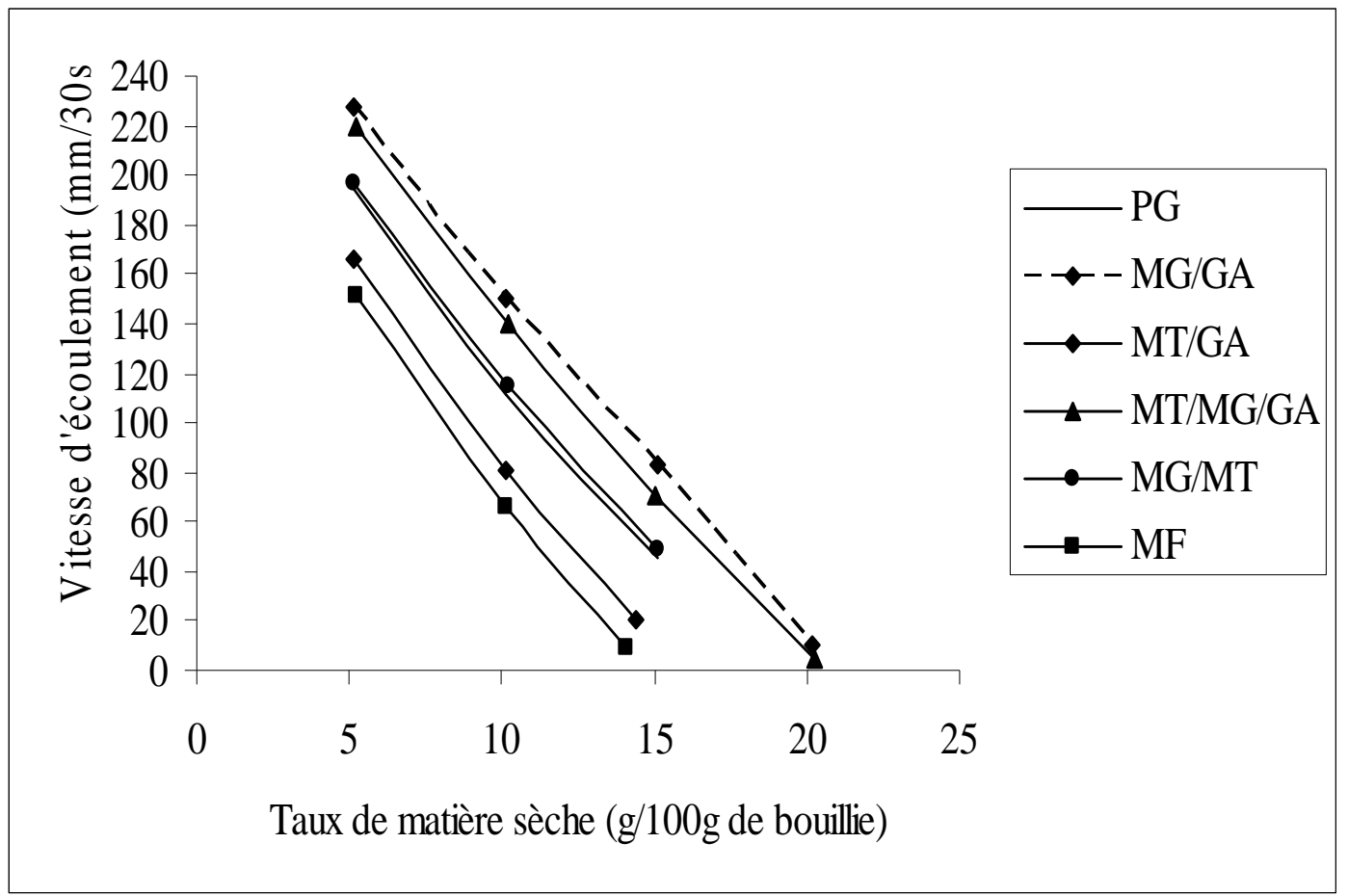

Figure 3 : Evolution de la vitesse d'écoulement des bouillies issues des procédés modifiés PG : bouillie à base de pâte de maïs germé MG/GA : bouillie à base de pâte de maïs germé - grains d'arachide MT/GA : bouillie à base de pâte de maïs trempé - grains d'arachide MT/MG/GA : bouillie à base de pâte de maïs trempé - maïs germé - grains d'arachide MT/MT : bouillie à base de pâte de maïs germé - maïs trempé MF : bouillie à base de pâte de maïs fermentée produite au laboratoire

La qualité nutritionnelle des pâtes et des bouillies (matière sèche, cendres, protéines, lipides) obtenues est identique à celle de l'ensemble du pays (Cornu et al., 1993) et à Brazzaville (Bouvier, 1992).

Les résultats obtenus montrent l'importance de l'association des céréales avec des légumineuses ou les protéoolagineux dans l'amélioration de l'état nutritionnel des enfants en période d'alimentation complétée. Ces résultats sont en accord avec ceux d'autres études antérieures qui ont ajouté des légumineuses dans la fabrication des aliments de complément à base de céréales (Tou (a) et al., 2007 ; Abiodun et al., 1999).
La moitié de la valeur protéique du maïs est composée de la zéine qui a une teneur très faible en deux acides aminés essentiels notamment le tryptophane $(80 \mathrm{mg} / 100 \mathrm{~g}$ de MS) et la lysine (331,4 mg/100 $\mathrm{g}$ de MS) et en niacine, vitamine du groupe $\mathrm{B}$ synthétisé à partir du tryptophane (Souci, 2000). Cette déficience peut être surmontée en ajoutant à celui-ci des légumineuses ou des protéooléagineux.

Les teneurs en protéines et en lipides des pâtes PG, MG/MT diminuent par rapport à celle de la pâte de maïs fermenté traditionnelle. Ainsi, notre étude n'a pas établi le rôle du maïs germé sur une quelconque augmentation de la teneur en protéines comme 
l'ont souligné certains travaux (Traoré et al., 2007).

Ces résultats obtenus montrent aussi une importante amélioration de la fluidité des bouillies dérivées de ces pâtes qui s'est traduite par un accroissement remarquable des vitesses d'écoulement au moins pour deux bouillies dérivées des pâtes issues des procédés modifiés notamment MG/GA, MT/MG/GA. Il ressort de ces résultats que les effets combinés de l'hydrolyse de l'amidon et des groupements polaires des acides gras de l'arachide se manifestent par l'augmentation de la vitesse d'écoulement des bouillies dérivées des pâtes améliorées de maïs fermenté comme le souligne Trèche (1996) et Tou (b) et al. (2007). Mais nos procédés n'ont pas permis de préparer des bouillies ayant un écoulement de $120 \mathrm{~mm} / 30$ s pour une teneur en matière sèche supérieure à $20 \mathrm{~g} / 100 \mathrm{~g}$ de bouillie (Dewey et Brown, 2003) à cause de l'acidité de la pâte conférée par la fermentation qui empêche l'amylase d'hydrolyser davantage l'amidon.

\section{Conclusion}

L'évaluation, dans le contexte de Pointe-Noire, de la qualité des bouillies traditionnelles dérivées des pâtes de maïs fermenté, utilisées comme aliment de complément du nourrisson et du jeune enfant, a montré que les procédés technologiques utilisés ont une incidence négative sur la valeur nutritionnelle et la densité énergétique de ces bouillies. La qualité nutritionnelle (protéines, lipides, cendres) de ces bouillies est quasiment identique à celle de l'ensemble du pays et à celle de la ville de Brazzaville.

Les modifications du procédé traditionnel de production de la pâte de maïs fermenté que nous avons introduites en réalisant la germination couplée à la fermentation en présence de farine humide de maïs germé et selon les cas, de grains d'arachide broyés, ont entraîné un accroissement important des teneurs en lipides et en protéines notamment pour les pâtes dont les grains d'arachide ont été incorporés. Cette augmentation des teneurs en nutriments s'est répercutée au niveau des bouillies dérivées de ces pâtes.

Ces modifications ont aussi induit d'importantes améliorations de la fluidité des bouillies dérivées de ces pâtes qui s'est traduite par un accroissement remarquable des vitesses d'écoulement au moins pour deux bouillies dérivées des pâtes issues des procédés modifiés notamment MG/GA, MT/MG/GA.

Toutefois, nos résultats ont été obtenus avec des vitesses d'écoulement de 120 $\mathrm{mm} / 30 \mathrm{~s}$ pour un taux de matière sèche inférieure à $15 \mathrm{~g} / 100 \mathrm{~g}$ de bouillie à cause de l'acidité conférée par la fermentation. Ces résultats sont intéressants et nous permettent d'envisager la poursuite de la mise au point des procédés qui permettront de produire des bouillies ayant $120 \mathrm{~mm} / 30 \mathrm{~s}$ pour une teneur en matière sèche de $20 \mathrm{~g} / 100 \mathrm{~g}$ de bouillie.

\section{REFERENCES BIBLIOGRAPHIQUES}

Abiodun IS, Anthony AO, Omolara TI. 1999. Biochemical composition of infant weaning food fabricated from fermented blends of cereal and soybean. Food Chemistry, 65: 35-39.

AOAC (Association of Official Analytical Chemists). 1980. Official Methods of Analysis (11th edn). AOAC: Washington, DC.

Bouvier M. 1992. Etude du mode de fonctionnement des ateliers de fabrication du «poto-poto » à Brazzaville. Mémoire de DESS, Université de Montpellier II, 48p.

Caulfield, Huffman SI, Piwoz EG. 1999. Interventions to improve intake of complementary foods by infants 6 to 12 months of age in developing countries impact on growth and on the prevalence 
of malnutrition and potential contribution to child survival. Food Nutr. Bull., 20: 183-200.

Cornu A, Trèche S, Massambba JP, Delpeuch F. 1993. Alimentation de sevrage et interventions nutritionnelles au Congo. Cahiers Santé, 3: 168-177.

Dewey KG, Brown KH. 2003. Update on technical issues concerning complementary feeding of young children in developing countries and applications for intervention programs. Food Nutr. Bull., 24(1): 5-28.

EDS-Congo. 2005. Ministère du Plan, de l'Aménagement du territoire, de l'Intégration économique et du NEPAD de la République du Congo (EDSCI), 35p Site internet: http//www.cnsee. org/Données/enquete/PDF/Congo\%20ED SC-1.pdf

Louembé D, Brauman A, Tchicaya F, Kobawila SC. 1996. Etude microbiologique et biochimique de la bouillie de maïs «poto-poto». Microbiologie-Aliments Nutrition, 14: 245-253.

Louembé D, Keleké S, Kobawila SC. 2003. Bactéries lactiques de la pâte fermentée de maïs. Tropicultura, 21(1): 3-9.

Louembé D, Kéléké S, Kobawila SC, Nzoussi JP. 2004. Variabilité et amélioration de la technologie traditionnelle de production de la pâte fermentée de maïs au Congo. Tropicultura, 22(4): 211-218.

Mensah P. 1997. Fermentation, the key ton food safety assurance in Africa ? Food Control, 8: 271-278.

Mouquet C, Trèche S. 2001. Viscosity of grues for infants a comparaison of measurement procedures. Int. J. Food Sci. Nut., 52: 389-400.

Mouquet C, Salvignol B, Van Hoan N, Monvois J, Trèche S. 2003. Ability of a very low cost extruder to produce instant infant flours at a small scale in Vietnam. Food Chemistry, 82: 249-255.
Muyanja CMBK, Narvhus JA, Treimo J, Langsrud T. 2002. Isolation, characterization and identification of lactic acid bacteria from bushera : a ugandan traditional fermented beverage. International Journal of Food Microbiology, 80: 201-210.

N'goma M'foundou RS. 2001. Etude des modalités d'utilisation des aliments de complément che le nourrisson et le jeune enfant de la ville de Pointe-Noire. Mémoire de fin d'études d'ingénieur, I.D.R., Université Marien NGOUABI, $56 \mathrm{p}$.

Nout MJR, Kok B, Vela E, Nche PE, Roumbouts FM. 1996. Acceleration of the fermentation of kenkey, an andigenous fermented maize food of Ghana. Food Resaerch International, 28(6): 599-604.

Oyewole Olusola B. 1997. Lactic fermented foods in Africa and their bebefits. Food Control, 8: 288-297.

Sanchez-Grinan Mi, Peerson J, Brown KH. 1992. Effect of dietary energy density on total ad libitum energy consumption by recovering malnourished children. Eur. J. Clin. Nutr., 46: 197-204.

Souci SW, Fachmann W, Kraut H. 2000. La Composition des Aliments. Tableaux des Valeurs Nutritives (6 $6^{\text {ème }}$ édn). CRC Press : Boca Raton ; 1182.

Tchibindat F, Malalou, Martin-Prevel, Gnimi, Ambendet, 1999. Enquête nutritionnelle, "Situation nutritionnelle dans la région du Kouilou”, Unicef congo, Urnah, 28p.

Thomazic M. 2003. Caractérisation et de la vente de «poto-poto». Mémoire de DESS, Université de Montpellier II, 68p.

Tou EH, Guyot JP, Mouquet-Rivier C, Rochette I, Counil E, Traoré AS, Trèche S. 2006. Study through surveys and fermentation kinetics of the traditional processing of pearl millet (Penissetum glaucum) into ben-saalga, a fermented gruel from Burkina Faso. International 
Journal of Food Microbiology, 106: 5260.

Tou (a) EH, Mouquet-Rivier C, Picq C, Traoré AS, Trèche S, Guyot JP. 2007. Improving the nutritional quality of bensaalga, a traditional fermented millet based gruel, by co-fermentig millet with groundnut and modifying the processing method. LWT, 40: 1561-1569.

Tou (b) EH, Mouquet-Rivier C, Rochette I, Traoré AS, Trèche S. 2007. Effect of different process combinaisons and energy of ben-saalga, a fermented gruel from Burkina Faso. Food Chemistry, 100: 935-943.
Traoré $\mathrm{T}$, Mouquet $\mathrm{C}$, Picq $\mathrm{C}$, Icard-Vernière C, Rochelle I, Traoré AS, Trèche S. 2007. Influence of technological know-how of producers on the biochemical characteristics of red sorghum malt from small scale production units in Ouagadougou (Burkina Faso). Journal of Food Sciences, 58(1): 63-76.

Trèche S. 1996. Influence de la densité énergétique et de la viscosité sur l'ingéré énergétique des nourrissons. Cahiers Santé, 6: 237-243.

Trèche. 2004. Les bouillies fluides, bébés bien nourris. Information pour le développement agricole des pays ACP, $\mathrm{N}^{\circ} 110$. 